\title{
Thermodynamic basis of the $a$-helix and DNA duplex
}

\author{
A. I. Dragan ${ }^{1} \cdot$ C. Crane-Robinson ${ }^{2}$ - P. P. L. Privalov ${ }^{3}$
}

Received: 26 December 2020 / Accepted: 20 March 2021 / Published online: 24 April 2021

(c) The Author(s) 2021

\begin{abstract}
Analysis of calorimetric and crystallographic information shows that the $\alpha$-helix is maintained not only by the hydrogen bonds between its polar peptide groups, as originally supposed, but also by van der Waals interactions between tightly packed apolar groups in the interior of the helix. These apolar contacts are responsible for about $60 \%$ of the forces stabilizing the folded conformation of the $\alpha$-helix and their exposure to water on unfolding results in the observed heat capacity increment, i.e. the temperature dependence of the melting enthalpy. The folding process is also favoured by an entropy increase resulting from the release of water from the peptide groups. A similar situation holds for the DNA double helix: calorimetry shows that the hydrogen bonding between conjugate base pairs provides a purely entropic contribution of about $40 \%$ to the Gibbs energy while the enthalpic van der Waals interactions between the tightly packed apolar parts of the base pairs provide the remaining $60 \%$. Despite very different structures, the thermodynamic basis of $\alpha$-helix and B-form duplex stability are strikingly similar. The general conclusion follows that the stability of protein folds is primarily dependent on internal atomic close contacts rather than the hydrogen bonds they contain.
\end{abstract}

Keywords $\alpha$-Helix $\cdot$ DNA double helix $\cdot$ Stability $\cdot$ Van der Waals interactions $\cdot$ Hydrogen bonding

\section{Introduction}

The first regular conformation of the polypeptide chain to be recognised, the $\alpha$-helix, was proposed by Pauling et al. (1951) and it appeared to be held together largely by hydrogen bonds resulting from association of the positively charged amino hydrogen of the $N$ th residue with the negatively charged carbonyl oxygen of the $(N+4)$ th residue along the polypeptide chain. In the less stable П-helix, the hydrogen bond connects with the $(N+5)$ th residue, with a resulting central axial space (see Fig. 1). In the disordered state of the polypeptide chain, the groups participating in this intramolecular H-bond are expected to be hydrogen bonded

P. L. Privalov: Deceased.

$\triangle$ C. Crane-Robinson

colyn.crane-robinson@port.ac.uk

1 Institute of High Technologies, Taras Shevchenko National University of Kyiv, Kyiv 01601, Ukraine

2 Biophysics Laboratories, School of Biology, University of Portsmouth, Portsmouth PO1 2DT, UK

3 Department of Biology, Johns Hopkins University, Baltimore, MD 21218, USA to water molecules, so it was difficult to envisage the source of any enthalpic stabilization of the helix from H-bonding. In contrast, formation of the helix must be accompanied by the shedding of water molecules from the amino and carbonyl groups into the bulk solvent, resulting in a substantial entropy increase. So what is the evidence that-in aqueous solution-intramolecular hydrogen bonds are entropic rather than enthalpic interactions?

With the definition of the DNA double helix by Watson and Crick (1953), it appeared that its regular structure is also maintained by hydrogen bonding, in this case between the conjugate base pairs: A with $\mathrm{T}$ and $\mathrm{G}$ with $\mathrm{C}$ (see Fig. 1). To investigate the physical basis of these two regular conformations, the $\alpha$-helix of polypeptide chains and the DNA duplex, various physical methods have been used: optical, hydrodynamic, crystallographic and scanning microcalorimetry (DSC), the last specially developed for this purpose (see Privalov 2012 for an overview). Importantly, DSC shows that heating of both the $\alpha$-helix and the DNA double helix results in melting their regular conformation and this proceeds with substantial heat absorption accompanied by a significant heat capacity increment. To make comparison between the two structures, the thermodynamic signatures of both the $\alpha$-helix and the DNA duplex need to be defined. 


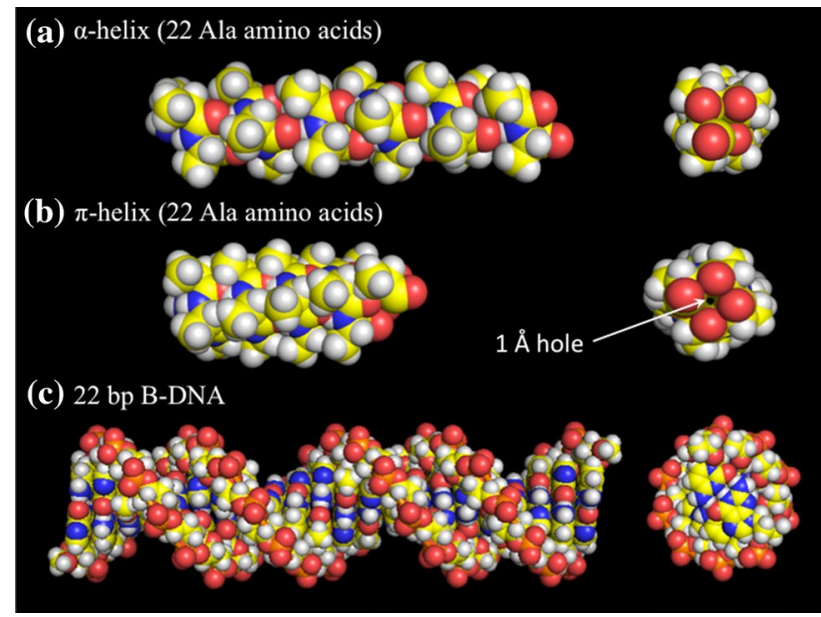

Fig. 1 Space filling models of the $\alpha$-helix, $\Pi$-helix and B-form DNA duplex. Only for the $\Pi$-helix is there any central space remaining in the structure

\section{Calorimetry of the $a$-helix}

Early studies of the $\alpha$-helix were carried out using isolated single helices from sperm whale myoglobin. However, the temperature induced unfolding of such helices is not a twostate transition because upon heating they gradually melt from both ends. Therefore, in our experiments, we used a single helix from the basic segment of the yeast bZIP protein GCN4, having covalently closed terminal loops attached at each end to block end-fraying (Taylor et al. 1999; Dragan et al. 2004a). This polypeptide is $100 \%$ helical and unfolds/ refolds in an all-or-nothing fully reversible manner, absorbing/releasing considerable heat (Fig. 2). Modelling the $C_{\mathrm{p}} / T$ function for melting this 29 amino acid polypeptide (the green curve in Fig. 2) as a 2-state process shows the best fit is for an enthalpy, $\Delta H$, of $69 \mathrm{~kJ} / \mathrm{mol}$-peptide and a heat capacity increase, $\Delta C_{\mathrm{p}}$, of $0.46 \mathrm{~kJ} / \mathrm{K}$.mol-peptide, i.e. $\Delta H=2.4 \mathrm{~kJ} / \mathrm{mol}$-amino acid and $\Delta C_{\mathrm{p}}=0.016 \mathrm{~kJ} / \mathrm{K}$. mol-amino acid. Other studies of individual $\alpha$-helices (e.g. Richardson et al. 1999; Lopez et al. 2002) led to somewhat higher enthalpy values of $\sim 3.5 \mathrm{~kJ} / \mathrm{mol}$-amino acid but no increase in heat capacity was observed. Calorimetric data obtained for $\alpha$-helices raise the question as to the source of the enthalpy and its temperature dependence, as well as the basis of its thermodynamic stability.

\section{Analysis of structural data for a-helices}

Unfolding of an $\alpha$-helix results in exposure of apolar and polar groups and this can give rise to a heat capacity change in consequence of altered hydration, i.e. an enthalpy dependence on temperature. The increase in accessible surface area, $\triangle$ ASA, of both polar and apolar surface, that becomes exposed on unfolding several $\alpha$-helices was determined using the NACCESS programme and these increases then used to predict the consequent heat capacity change, $\Delta C_{\mathrm{p}}$, calculated using the equation proposed by Makhatadze and Privalov (1995):

$\Delta C_{\mathrm{p}}=2.14 \times \Delta \mathrm{ASA}^{\mathrm{apolar}}-1.27 \times \Delta \mathrm{ASA}^{\text {polar }}$,

where $\Delta$ ASA values are in $\AA^{2}$ and the coefficients typically in $\mathrm{J} / \mathrm{K} / \AA^{2}$.

This equation reflects the fact that exposure of apolar surface-meaning its hydration-leads to an increase in the heat capacity (positive $\Delta C_{\mathrm{p}}$ ), whereas exposure of polar surface results in a decreased heat capacity (negative $\Delta C_{\mathrm{p}}$ ).

The first entry in Table 1 gives data for a fully folded 'all- $\alpha$ ' protein: sperm whale myoglobin (swMb). It consists of 8 tightly packed helices and the interior is very rich in non-polar contacts-relative to polar contacts-with the result that the heat capacity increases very substantially on melting. The predicted $\Delta C_{\mathrm{p}}$ is in good agreement with
Fig. 2 The partial specific heat capacity functions of the isolated basic segment of GCN4 with (1) free ends, red line; (2) attached at the C-terminal end to the leucine zipper, blue line (Dragan et al. 2004a); (3) the basic segment with two covalently closed terminal loops, green line (Taylor et al. 1999)

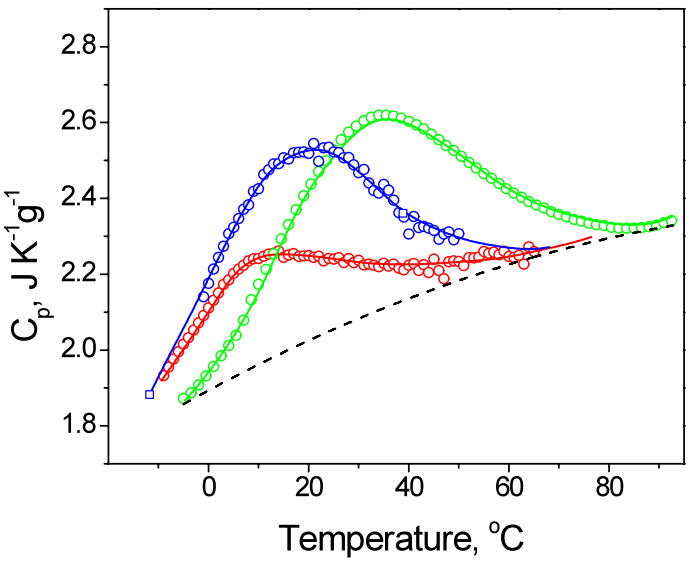

(1) Free $\mathrm{N}$ - and C-terminal ends

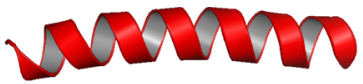

(2) Free $\mathrm{N}$ - and closed C-terminal end

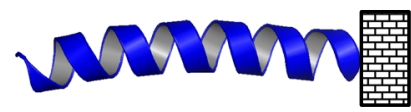

(3) Closed N- and C-terminal ends

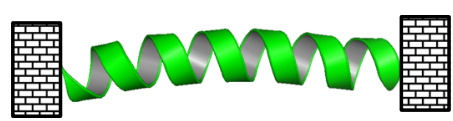


Table 1 Analysis of changes in solvent accessible areas, ASAs, (polar and apolar), upon unfolding an intact protein and three alpha-helices

\begin{tabular}{|c|c|c|c|c|c|c|c|c|}
\hline \multirow[t]{2}{*}{ Object } & \multirow{2}{*}{$\begin{array}{l}\text { Polarity } \\
\text { ASA polarity }\end{array}$} & \multicolumn{5}{|l|}{ Calculated data } & \multicolumn{2}{|c|}{ Experimental data } \\
\hline & & $\begin{array}{l}\text { ASA (folded) } \\
\AA^{2}\end{array}$ & $\begin{array}{l}\text { ASA } \\
\text { (unfolded) } \\
\AA^{2}\end{array}$ & $\Delta \mathrm{ASA} \AA^{2}$ & $\begin{array}{l}\text { Overall } \\
\Delta C_{\mathrm{p}}, \mathrm{kJ} / \\
(\mathrm{K} . \mathrm{mol})\end{array}$ & $\begin{array}{l}\Delta C_{\mathrm{p}}, \mathrm{J} / \\
\text { (K.mol- } \\
\text { residue) }\end{array}$ & $\begin{array}{l}\text { Overall } \\
\Delta C_{\mathrm{p}}, \mathrm{kJ} / \\
(\mathrm{K} . \mathrm{mol})\end{array}$ & $\begin{array}{l}\Delta C_{\mathrm{p}}, \mathrm{J} / \\
\text { (K.mol- } \\
\text { residue) }\end{array}$ \\
\hline \multirow{2}{*}{$\begin{array}{l}\text { swMb: intact sperm whale myo- } \\
\text { globin, } 153 \text { aa }\end{array}$} & Apolar & 4928 & 13,110 & 8182 & 14.4 & 93.5 & 14 & 93 \\
\hline & Polar & 2980 & 5431 & 2451 & & & & \\
\hline \multirow{2}{*}{$\begin{array}{l}\alpha \text {-Helix generated from complete } \\
\text { swMb sequence }\end{array}$} & Apolar & 10,853 & 13,110 & 2257 & 1.92 & 12.47 & NK & NK \\
\hline & Polar & 3140 & 5431 & 2291 & & & & \\
\hline \multirow{2}{*}{$\begin{array}{l}\alpha \text {-Helix from the } 29 \text { a.a. GCN4 } \\
\text { sequence from Taylor et al. } \\
\text { (1999) }\end{array}$} & Apolar & 1246 & 1622 & 376 & 0.24 & 12.7 & 0.46 & 16 \\
\hline & Polar & 908 & 1350 & 442 & & & & \\
\hline \multirow{2}{*}{$\begin{array}{l}\alpha \text {-Helix from the Lpp56 sequence } \\
\text { Dragan et al. }(2004 b)\end{array}$} & Apolar & 3567.4 & 4707.4 & 1140 & 0.94 & 16.78 & 0.83 & 14.8 \\
\hline & Polar & 1629.2 & 2810.3 & 1181 & & & & \\
\hline
\end{tabular}

Calculation of corresponding unfolding heat capacities and comparison with experimental data

Heat capacity changes calculated using Eq. 1 (see Makhatadze and Privalov 1995) based on changes in Accessible Surface Area ( $\Delta$ ASA), obtained using NACCESS, for a single intact protein (sperm whale myoglobin) and three $\alpha$-helices generated in silico

NKnot known

the experimentally measured value of $93 \mathrm{~J} / \mathrm{K}$.mol-residue, indicating the reliability of Eq. (1).

The remaining three entries in Table 1 are individual helices with $\alpha$-helical coordinates generated in silico. The first is the complete sequence of swMb configured as one continuous $\alpha$-helix, the second is the fully helical 29 residue monomeric polypeptide studied calorimetrically by Taylor et al. (1999) and the third is the 56 residue sequence of Lpp56, the unit of the trimeric $\alpha$-helical coiled-coil from the outer membrane of E. coli, studied by Dragan et al. (2004b). For all three $\alpha$-helices unfolding leads to exposure of similar total areas per amino acid, amounting to $\sim 30 \AA^{2}$. Furthermore, the areas of apolar and polar surface exposed are approximately equal and since the apolar coefficient in Eq. (1) is significantly greater than the polar coefficient, the prediction is a net positive value of $\Delta \mathrm{Cp}$, calculated as +(12-17) J/K.molresidue for these three helices. Strikingly, these values are close to those measured experimentally for the GCN4derived polypeptide of Taylor et al. (1999), $\Delta C_{\mathrm{p}}=16 \mathrm{~J} / \mathrm{K}$. mol-residue, and the Lpp56 monomer from Dragan et al. (2004b), $\Delta C_{\mathrm{p}}=14.8 \mathrm{~J} / \mathrm{K} . \mathrm{mol}-$ residue. The modelling of these $\alpha$-helices in silico therefore gives a good representation of their structure in solution and provides an understanding of why the heat capacity change on melting the $\alpha$-helix has a small positive value.

The dominance of the first term of Eq. (1) shows that the van der Waals interactions between tightly compacted apolar atoms are the prime contributor to $\alpha$-helix stabilization. If the H-bonds were the principle agent of helix stability then the exposure of their polar surface would be expected to dominate $\triangle \mathrm{ASA}$ and the heat capacity change on unfolding consequently be negative. But this is not observed: the heat capacity change is positive, i.e. the stabilizing enthalpy comes principally from the hydrophobic interactions.

\section{Comparing the thermodynamic signatures of the $a$-helix and the DNA duplex}

The substantial heat effect $(\Delta \mathrm{H})$ and its significant dependence on temperature $(\Delta \mathrm{Cp})$ for melting the DNA duplex have recently been investigated in detail using short synthetic duplexes (Vaitiekunas et al. 2015; Dragan et al. 2019; Privalov and Crane-Robinson 2020), see Fig. 3. This allowed

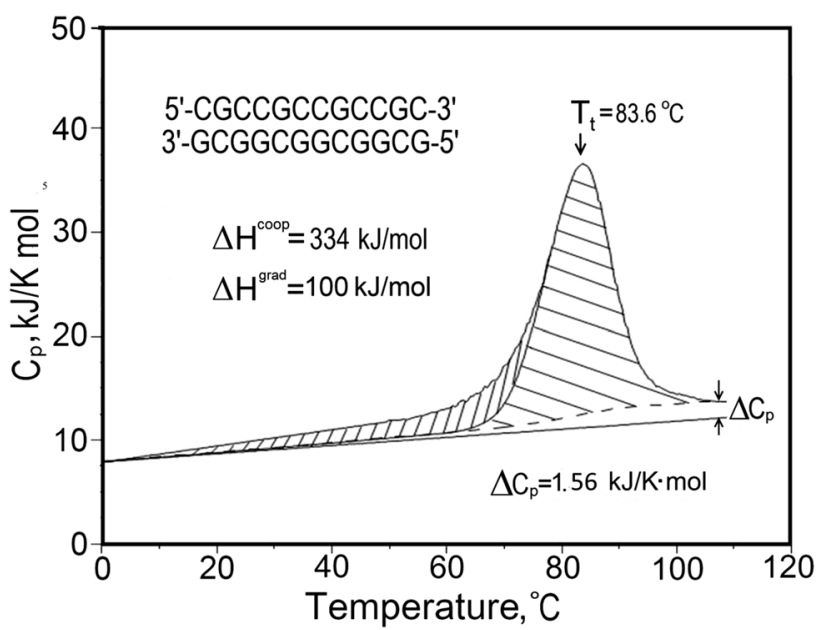

Fig. 3 The observed heat capacity profile of a $12 \mathrm{bp}$ all-CG duplex. The experimental excess heat effect is deconvoluted into the noncooperative (gradual, vertical hatching) and cooperative (horizontal hatching) phases (see Vaitiekunas et al. 2015) 
determination of the thermodynamic parameters characteristic of the dissociation of CG and AT pairs. After making allowance for the loss of minor groove hydration to the AT pairs, it was found that the intrinsic melting enthalpy of both pairs is essentially the same. Since the CG pair carries an extra $\mathrm{H}$-bond relative to AT, it follows that $\mathrm{H}$-bonding is not contributing to the observed enthalpies. As regards the overall entropy of dissociation, which has a large positive conformational contribution, the more positive net value observed for the AT pair (by $4 \mathrm{~J} / \mathrm{K}$.mol-bp) reflects a lower negative contribution to $\Delta S$ from AT hydration as compared to CG hydration: $-4 \mathrm{~J} / \mathrm{K}$.mol-bp is thus the entropy decrease from hydration on dissociating a single $\mathrm{H}$-bond.

The observation that dissociation of $\mathrm{H}$-bonds in DNA melting is non-enthalpic accords with the expectation that the process is an exchange of H-bonding, not just the loss of base pairing. Moreover, the purely entropic nature of H-bond disruption-in water solution-is not unexpected as H-bonds are essentially electrostatic contacts, interactions experimentally demonstrated to be entirely entropic. For example, in protein/DNA complexes the ionic binding of negative phosphates and positive sidechains of lysine and arginine means that the affinity is strongly reduced by increased ionic strength. However, it is observed that the enthalpy of the protein/DNA interaction is independent of the salt concentration (see Dragan et al. 2004c; Privalov et al. 2011), showing that the ionic links are indeed nonenthalpic. The drop in affinity with increasing ionic strength results entirely from the entropy of released counter-ions (principally cations from the DNA) being less at higher salt concentrations.

The conclusion therefore is that the contribution of H-bond pairing to DNA duplex stability is entirely entropic in nature, i.e. non-enthalpic. So what is the source of the calorimetrically observed enthalpy? The answer can only be that $\Pi-\Pi$ stacking of base pairs is the main contributor, as expected from the observation of substantial enthalpy on melting single stranded oligo-A, a situation in which no internal H-bonding is expected (Breslauer and Sturtevant 1977).
Although both the $\alpha$-helix and the duplex are rod-like macromolecules, the question arises as to whether they are supported by a similar combination of thermodynamic forces. In both cases, there is a substantial negative enthalpy driving formation of the folded structures and this is enhanced by the positive entropy derived from dehydrating the polar groups that form the H-bonds. In structural terms, base stacking in the duplex and close packing of atoms in the $\alpha$-helix provide the driving enthalpy, while a purely entropic force is provided by the release of water molecules from the DNA bases and from the peptide atoms that form $\mathrm{H}$-bonds in the $\alpha$-helix. These two driving forces are, of course, opposed by the substantial reduction in conformational entropy on forming both structures.

Table 2 compares the key thermodynamic parameters for the base pairs of DNA with those determined for the 29 -residue $\alpha$-helix derived from GCN4. The data for the AT and CG pairs of DNA are taken from Privalov and CraneRobinson 2020 and for the $\alpha$-helix the 29-residue polypeptide of Taylor et al. 1999, all at the standard temperature of $25^{\circ} \mathrm{C}$-which is close to the melting temperature of this helix $\left(T_{\mathrm{m}}=28^{\circ} \mathrm{C}\right)$. Importantly, it is assumed that the thermodynamic parameters for forming the $\mathrm{H}$-bond in the $\alpha$-helix are the same as for the intermolecular H-bonds in the DNA duplex, i.e. non-enthalpic with an entropy increase of $4 \mathrm{~J} / \mathrm{K} . \mathrm{mol} \mathrm{H}$-bond, which provides an entropy factor $(\Delta T \Delta S)$ of $-1.2 \mathrm{~kJ} / \mathrm{mol} \mathrm{H}$-bond to the overall Gibbs free energy.

The data in Table 2 show that the enthalpy/entropy pattern is similar for formation of the two structures. As illustrated in Fig. 4, the enthalpy is the dominant driving force (in red), counteracted by the large reduction in conformational entropy (in cyan) but the entropy increase from water release on forming the $\mathrm{H}$-bonds (dark blue) makes a favourable contribution to folding both the duplex and the helix.

The last two rows of Table 2 quantify the relative contribution of entropic $\mathrm{H}$-bonding to the overall stability of the two macromolecules. The first $\left(T \Delta S^{\mathrm{H} \text {-bonding } / \Delta G)}\right.$ is the $\mathrm{H}$-bonding entropy factor as a proportion of the total Gibbs energy. This ratio is not meaningful for the $\alpha$-helix used here as its Gibbs energy is so close to zero at $25^{\circ} \mathrm{C}$, but for the
Table 2 Data for formation of the DNA duplex (Privalov and Crane-Robinson 2020) and for folding a 29 amino acid $\alpha$-helix derived from GCN4 (Taylor et al. 1999; Dragan et al. 2004a)

\begin{tabular}{|c|c|c|c|}
\hline All at $25^{\circ} \mathrm{C}$ & DNA & DNA & $\alpha$-HELIX \\
\hline$\Delta H \mathrm{~kJ} / \mathrm{mol}-\mathrm{bp}$ & -19 & -19 & - $2.31 \mathrm{~kJ} / \mathrm{mol} . \mathrm{a} . \mathrm{a}$. \\
\hline$\Delta S \mathrm{~J} / \mathrm{K} . \mathrm{mol}-\mathrm{bp}$ & -36.2 & -40.5 & - 7.82 J/K.mol.a.a. \\
\hline$-T \Delta S \mathrm{~kJ} / \mathrm{mol}-\mathrm{bp}$ & +10.7 & +11.9 & +2.33 kJ/mol.a.a. \\
\hline$\Delta G \mathrm{~kJ} / \mathrm{mol}-\mathrm{bp}$ & -8.3 & -7.1 & $+0.02 \mathrm{~kJ} / \mathrm{mol} . \mathrm{a} . \mathrm{a}$. \\
\hline$T \Delta S \mathrm{~kJ} / \mathrm{mol} \mathrm{H}$-bond (assumed constant) & +1.2 & +1.2 & $+1.2 \mathrm{~kJ} / \mathrm{mol} \mathrm{H}$-bond \\
\hline$-T \Delta S^{\mathrm{H}-\text { bonding }} \mathrm{kJ} / \mathrm{mol}-\mathrm{bp}$ (total) & -3.6 & -2.4 & $-1.2 \mathrm{~kJ} / \mathrm{mol} . \mathrm{a} . \mathrm{a}$. \\
\hline$-T \Delta S^{\text {Conformational }} \mathrm{kJ} / \mathrm{mol}-\mathrm{bp}$ & +14.3 & +14.4 & + 3.53 kJ/mol.a.a. \\
\hline$T \Delta S^{\mathrm{H}-\text { bonding }} / \Delta G$ & 0.43 & 0.34 & Not meaningful \\
\hline$T \Delta S^{\mathrm{H}-\text { bonding }} / \Delta H$ & 0.19 & 0.13 & 0.52 \\
\hline
\end{tabular}




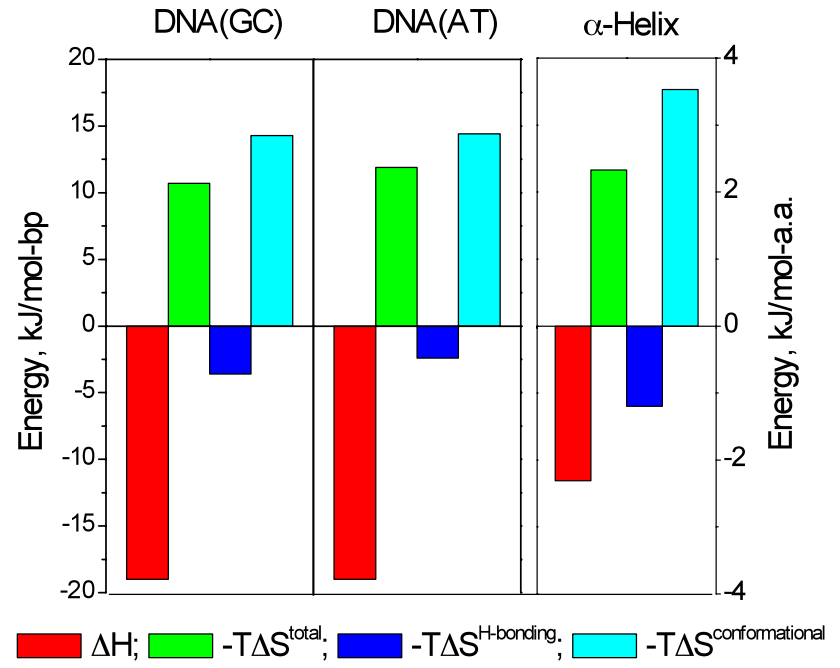

Fig. 4 Enthalpies and entropies of forming the base pairs of the DNA duplex and folding the $\alpha$-helix at $25{ }^{\circ} \mathrm{C}$. Enthalpies in red. The total entropy factor (in green) is made up of a large reduction in conformational entropy ( $T \Delta S^{\text {conformational }}$ in cyan) and an increase in entropy from water release on forming the H-bonds (in dark blue)

DNA base pairs, an averaged ratio of 0.4 implies that $40 \%$ of the Gibbs energy is entropic, i.e. $60 \%$ enthalpic.

The second ratio $\left(T \Delta S^{\mathrm{H}-\text { bonding }} / \Delta H\right)$ is the H-bonding entropy factor as a proportion of the enthalpy, the other component of the driving force for folding, seen in Fig. 4 as the blue relative to the red bars. This ratio shows that for the duplex the entropic free energy derived from water release on forming the base pairs represents about $15 \%$ of the enthalpic free energy derived from base stacking and other van der Waals close contacts. For the $\alpha$-helix, the H-bonding entropy factor gives about half as much free energy as does the enthalpic component derived from atomic close contacts, i.e. the $\mathrm{H}$-bonds provide approximately $1 / 3 \mathrm{rd}$ of the total force driving helix folding.

\section{Conclusions}

The data in Table 2/Fig. 4 demonstrate that the free energy driving folding of the $\alpha$-helix and the DNA duplex comes from two sources, the entropy of releasing water bound to the polar groups that form H-bonds, which is the minor component, and the majority component, which is enthalpic. Thus although the two macromolecules are very different in their molecular composition and structure, they are similar as regards the forces driving their formation. For both polymers, although the hydrogen bonds are responsible for defining the specificity of the structures, it is the van de Waals forces between closely packed atoms that play the primary role in maintaining their overall stabilities.
The finding that for the $\alpha$-helix-as with the DNA duplex - the entropy derived from forming internal H-bonds is of lower magnitude than the enthalpy, raises the question as to the overall role of $\mathrm{H}$-bonding in protein folding and structure. Hydrogen bonds are involved in secondary structure elements in addition to the $\alpha$-helix and in tertiary contacts, the 'dehydration entropy' of which should not differ greatly from that in the $\alpha$-helix. The overall picture is clear from the surface areas dehydrated on folding. Whereas for the isolated $\alpha$-helix the polar and apolar $\Delta$ ASAs are approximately equal, for completely folded domains such as swMb (see Table 1) $\triangle \mathrm{ASA}^{\text {apolar }}$ is very much greater than $\Delta \mathrm{ASA}^{\text {polar }}$, implying that the enthalpy of van der Waals contacts greatly exceeds the entropy factor derived from water release on forming H-bonds. This means that the concept of protein fold largely maintained by a network of H-bonds is not an appropriate model. The leading role in stabilizing protein folds is played by the van der Waals forces derived from internal close contacts.

Acknowledgements Initiation of this work was funded by the National Institutes of Health (NIH) Grant no. 105365 to P.L.P. and subsequently supported by the Ministry of Education and Science of Ukraine Grant no. 0116U004757/16БФ07-03 (to D.A.I.).

Open Access This article is licensed under a Creative Commons Attribution 4.0 International License, which permits use, sharing, adaptation, distribution and reproduction in any medium or format, as long as you give appropriate credit to the original author(s) and the source, provide a link to the Creative Commons licence, and indicate if changes were made. The images or other third party material in this article are included in the article's Creative Commons licence, unless indicated otherwise in a credit line to the material. If material is not included in the article's Creative Commons licence and your intended use is not permitted by statutory regulation or exceeds the permitted use, you will need to obtain permission directly from the copyright holder. To view a copy of this licence, visit http://creativecommons.org/licenses/by/4.0/.

\section{References}

Breslauer KJ, Sturtevant JM (1977) A calorimetric investigation of single stranded base stacking in the ribo-oligonucleotide A7. Biophys Chem 7:205-209

Dragan AI, Frank L, Liu Y, Makeyeva EN, Crane-Robinson C, Privalov PL (2004a) Thermodynamic signature of GCN4-bZIP binding to DNA indicates the role of water in discriminating between the AP-1 and ATF/CREB sites. J Mol Biol 343:865-878

Dragan AI, Potekhin SA, Sivolob A, Lu M, Privalov PL (2004b) Kinetics and thermodynamics of the unfolding and refolding of the three-stranded $\alpha$-helical coiled coil, Lpp-56. Biochemistry 43:14891-14900

Dragan AI, Read CM, Makeyeva EN, Milgotina EI, Churchill ME, Crane-Robinson C, Privalov PL (2004c) DNA binding and bending by HMG boxes: energetic determinants of specificity. J Mol Biol 343:371-393

Dragan A, Privalov PL, Crane-Robinson C (2019) Thermodynamics of DNA: heat capacity changes on duplex unfolding. Eur Biophys J 48:773-779 
Lopez MM, Chin D-H, Baldwin RL, Makhatadze GI (2002) The enthalpy of the alanine peptide helix measured by isothermal titration calorimetry using metal-binding to induce helix formation. Proc Natl Acad Sci USA 99:1298-1302

Makhatadze GI, Privalov PL (1995) Energetics of protein structure. Adv Prot Chem 47:307-425

Pauling LC, Corey RB, Branson HR (1951) The structure of proteins; two-hydrogen bonded helical configurations of the polypeptide chain. Proc Nat Sci USA 37:205-211

Privalov PL (2012) Microcalorimetry of macromolecules. Wiley

Privalov PL, Crane-Robinson C (2020) Forces maintaining the DNA double helix. Eur Biophys J 49:315-321

Privalov PL, Dragan AI, Crane-Robinson C (2011) Interpreting protein/ DNA interactions: distinguishing specific from non-specific and electrostatic from non-electrostatic components. Nucleic Acids Res 39:2483-2491

Richardson JM, McMahon KW, MacDonald CC, Makhatadze GI (1999) MEARA sequence repeat of human CstF-64 polyadenylation factor is helical in solution. A spectroscopic and calorimetric study. Biochemistry 38:12869-12875

Taylor JW, Greenfield NJ, Wu B, Privalov PL (1999) Calorimetric study of the folding-unfolding of an $\alpha$-helix with covalently closed $\mathrm{N}$ - and C-terminal loops. J Mol Biol 291:965-976

Vaitiekunas P, Crane-Robinson C, Privalov PL (2015) The energetic basis of the DNA double helix: a combined microcalorimetric approach. Nucleic Acids Res 43:8577-8589

Watson JD, Crick FHC (1953) Molecular structure of Nucleic acid. A structure for desoxyribonucleic acid. Nature 171:737-773

Publisher's Note Springer Nature remains neutral with regard to jurisdictional claims in published maps and institutional affiliations. 